\title{
Focus on spinal fractures in the elderly
}

\author{
H. Laurer · I. Marzi
}

Received: 31 July 2013/Accepted: 2 August 2013/Published online: 27 August 2013

(c) Springer-Verlag Berlin Heidelberg 2013

Indications for surgical intervention in the geriatric spine has been expanded over the last several decades, better meeting the needs of a growing elderly community with increased life expectations and activity level. In contrast to younger patients, injury in this age group is often caused by minor trauma facilitated from osteoporotic bone quality_one of the main factors responsible for increasing costs in any health system. Cement augmentation techniques have gained widespread use as the sole method to treat osteoporotic fractures, although important studies have opened up a broad discussion about indications and especially benefit for the patient. To that end, the paper from Benneker and Hoppe [1] gives a fresh update on this major tool in geriatric spine surgery.

Besides treating spontaneous fractures induced from poor bone quality with cement augmentation, this technique has been established more recently as a supportive tool in the treatment of trauma-induced spine fractures in the elderly. To date, cemented pedicle screws were used in patients that were subjected to dorsal instrumentation intended to increasing pull-out strength, which has been shown in the preclinical testing. Especially the use of cannulated and fenestrated screws to become cemented after insertion raises the opportunity to reduce hardware failure, as reported by El Saman et al. [2].

The possibilities for complications using cement injection into the vertebrae are abundant, beginning with minor local

\footnotetext{
H. Laurer $(\varangle) \cdot$ I. Marzi
}

Department of Trauma, Hand, and Reconstructive Surgery,

University Hospital, Goethe-University Frankfurt,

Theodor-Stern-Kai 7, 60590 Frankfurt am Main, Germany

e-mail: laurer@trauma.uni-frankfurt.de

URL: www.trauma.uni-frankfurt.de

I. Marzi

e-mail: marzi@trauma.uni-frankfurt.de extrusions into the surrounding tissue, which, in general, are not harmful to the patient. However, reports of severe side effects like pulmonary embolism of cement requires new awareness of the complications as well as ideas and techniques designed to overcome these issues in the future. The innovative and promising observation that increasing PEEP during cement application might be beneficial is reported by El Saman et al. [3]. Hopefully, this paper will give inspiration to numerous other investigations intended to continue the amelioration of cement usage in spine surgery.

Despite this tremendous progress in surgical treatment options and intensive care medicine injury, especially the cervical spine in the geriatric population is associated with high mortality in the hospital and extending up to 3 months after trauma, as shown by Sander et al. [4]. Moreover, as the outcome is mainly influenced by the neurological deficit upon admission, "do not further harm" seems to be the optimal treatment concept-stressing the utmost importance of qualified physicians able to read the radiographs taken. Particularly, since incidental findings increase with age, profound knowledge of these variations investigated by Koren and colleagues [5] and also reported in this issue seem mandatory.

Taken together, the critical assessment of technical developments in combination with continuing staff training might help to achieve an individualized treatment algorithm for these elderly patients and indicate the best treatment available.

Conflict of interest None.

\section{References}

1. Benneker LM, Hoppe S. Percutaneous cement augmentation techniques for osteoporotic spinal fractures. Eur J Trauma Emerg Surg 2013. doi:10.1007/s00068-013-0265-7 
2. El Saman A, Meier S, Sander A, Kelm A, Marzi I, Laurer H. Reduced loosening rate and loss of correction following posterior stabilization with or without PMMA augmentation of pedicle screws in vertebral fractures in the elderly. Eur J Trauma Emerg Surg 2013. doi:10.1007/s00068-013-0310-6

3. El Saman A, Kelm A, Meier S, Sander AL, Eichler K, Marzi I, et al. Intraoperative PEEP-ventilation during PMMA-injection for augmented pedicle screws: improvement of leakage rate in spinal surgery. Eur J Trauma Emerg Surg 2013. doi:10.1007/s00068-0130319-x
4. Sander Al, El Saman A, Delfosse P, Wutzler S, Meier S, Marzi I, et al. Cervical spine fractures in the elderly: morbidity and mortality after operative treatment. Eur J Trauma Emerg Surg 2013. doi:10.1007/s00068-013-0311-5

5. Koren L, Simonovich A, Norman D, Israelit S, Jerdev M, Shreter $\mathrm{R}$, et al. Do cervical spine $\mathrm{X}$-rays for trauma have clinically significant incidental findings? Eur J Trauma Emerg Surg 2013. doi:10.1007/s00068-013-0290-6 